\title{
Market-based vs. accounting-based performance of banks in Asian emerging markets
}

\author{
Li Li \\ School of Business, University of the Thai Chamber of Commerce
}

\begin{abstract}
This paper compares the market-based and accounting-based performances of listed commercial banks among eight Asian emerging markets such as China, India, Indonesia, Korea, Malaysia, the Philippines, Taiwan and Thailand over the period 2005-2010. Marketbased performance is measured by Tobin's Q ratio and accounting-based performance is measured by three conventional ratios for a bank: net interest margin, return on assets and return on equity. It is found that Indonesian banks have the highest average Tobin's Q of 1.14, return on assets of 1.82 percent and net interest margin of 5.34 percent, whereas banks in China and India have the highest average return on equity of 18.22 percent. Banks in Korea, Taiwan and Thailand have the lowest average Q ratio of 1.00. Banks in Taiwan also have the lowest average return on assets of negative 0.57 percent, return on equity of negative 7.80 percent and net interest margin of 1.97 percent. Q is shown to be significantly positively related to return on assets and return on equity, but there is no significant relationship between $\mathrm{Q}$ and net interest margin. Further, the determinants of each type of performance are found to be different. The GLS regression result reveals that return on assets is significantly positively associated to the bank's capital adequacy; return on equity is significantly positively linked to asset quality; net interest margin is significantly positively affected by capital adequacy and negatively affected by liquidity; whereas the Q ratio is significantly positively related to capital adequacy and asset quality. Finally, the different regression results for banks in each single emerging market indicate that the market-based and accounting-based performances of commercial banks in different emerging market are affected by different factors in different ways.
\end{abstract}

JEL classification: G2; G21

Key words: market-based, accounting-based, performance, banks, Asian emerging market

\section{Introduction}

The world economy and financial system have gone through a lot of changes during the last decade. The role of the emerging economies has been discussed more than ever after the recent U.S. economic crisis and European debt crisis. While China replaced Japan as the world's second-largest economy in 2010, it has been also said that the emerging markets will lead the world out of the recession triggered by the developed economies. Since the birth of the "emerging market" concept, it is very difficult to give an exact definition or make an 
exact list of emerging markets. Different lists from Morgan Stanley Capital International (MSCI), Standard and Poor's (S\&P), FTSE and The Economist, etc. consist of different countries. Thus, to limit the scope, this research intends to study the Asian emerging markets following the MSCI emerging market index, i.e. eight emerging markets included in the current study are: China, India, Indonesia, Korea, Malaysia, Philippines, Taiwan and Thailand.

Banking industry is no doubt the important sector of the economy, not to mention in the emerging markets. Further, the banking sector is always the first to be affected in the financial crisis, and it is always been heavily regulated and re-regulated. Hence, the performance monitoring of the banking sector is essential for all economies, especially in the emerging markets. Many research papers (e.g. Berger, Clarke, Cull, Klapper and Udell, 2005; Berger, Deyoung, Genay and Udell, 2000; Bonin, Hasan, and Wachtel, 2005; Jonghe and Vennet, 2008; Kosmidou and Zopounidis, 2008) study the performance of the banking sector from different aspects. The performance indicators can be classified into two categories such as accounting based and market based measures. Accounting based measures include the traditional ratios such as return on assets, return on equity, and net interest margin. Market based measures consist of stock return and Tobin's Q ratio. Therefore, this research will focus on these two types of performance measures to investigate the banking sector's performance in Asian emerging markets.

There are three objectives in this research: first, the paper intends to compare the accounting based and market based performances of banks in 8 Asian emerging markets; second, it will investigate the relationship between market based and accounting based performances; third, it will explore the determinants of market based and accounting based performances. The study period is chosen as 2005-2010. This period is chosen because these markets are emerging, hence the earlier data may not available or complete.

The rest of the study is organized as follows: the second part will review the literature; the third part will illustrate the data and methodology; and the fourth part explains the results of the study and the last part of the paper concludes.

\section{Literature review}

Market based performance is commonly measured by variables such as stock return and Tobin's Q. Choi and Hasan (2005) use the annual stock return and the standard deviation of the daily stock returns to measure the market based performances, return and risk, of Korean commercial bank industry over the period 1998-2002. Chunhachinda and Jumreornvong (1999) use the Tobin's Q to measure the competitiveness of banks and finance companies in Thailand over the period 1990-1996. Chunhachinda and Li (2011) employ Tobin's Q to measure and compare the competitiveness of Asian banks after recovering from the 1997 financial crisis. Jonghe and Vennet (2008) apply the Tobin's Q to measure the European banks' franchise value. Jones, Miller and Yeager (2011) utilize the Tobin's Q to proxy for the charter value in the banking industry.

Accounting based performance is more measured by conventional ratios for a bank such as net interest margin (NIM), return on assets (ROA) and return on equity (ROE) (Bonin, Hasan and Wachtel, 2005; Jonghe and Vennet, 2008; Kosmidou and Zopounidis, 2008; Lin and Zhang, 2009; Micco, Panizza and Yanez, 2007). 
The possible factors that may determine the market based and accounting based performance of banks are often chosen based on the widely used CAMEL (capital adequacy, asset quality, management quality, earnings, and liquidity) rating system such as in Kosmidou and Zopounidis (2008), Joen, Olivero and Wu (2010), Jones, Miller and Yeager (2010) and Chunhachinda and Li (2011). The effects of each factor on performance are not uniform across the studies.

To the author's best knowledge, there is no paper that has compared the accounting based and market based performances (and their determinants) of 8 Asian emerging markets or studied the relationship between the two. Hence, this study contributes to the literature on empirical results of the accounting based and market based performances of 8 Asian emerging markets.

\section{Data and Methodology}

\section{Data}

The study focuses on the comparison of the market based and accounting based performances of banks among eight Asian emerging markets (China, India, Indonesia, Korea, Malaysia, Philippines, Taiwan and Thailand) over the period 2005-2010. Therefore, only commercial banks (excluding financial holdings companies, investment banks, securities and finance companies, cooperatives and Islamic banks, etc) listed on the stock exchanges will be included in the sample and the bank observations that market stock prices are not available will be deleted. All annual accounting and market data of listed commercial banks of the eight emerging Asian markets are compiled from Bankscope. The final unbalanced panel sample consists of 102 banks and 470 bank-year observations: 16 banks and 64 bank-year observations from China, 6 banks and 29 bank-year observations from India, 25 banks and 133 bank-year observations from Indonesia, 13 banks and 41 bank-year observations from Korea, 3 banks and 13 bank-year observations from Malaysia, 15 banks and 72 bank-year observations from Philippines, 12 banks and 61 bank-year observations from Taiwan and 12 banks and 57 bank-year observations from Thailand.

\section{Methodology}

To achieve the three objectives of the study, the data will be analyzed as follows:

1) To compare the accounting based and market based performances of banks in 8 Asian emerging markets: the following data and performance measures are compiled, calculated and compared:

$\mathrm{ROA}=$ net income/average total assets

$\mathrm{ROE}=$ net income/average total equity

$\mathrm{NIM}=$ net interest and dividend income/average total earning assets

$\mathrm{Q}=$ (market capitalization + book value of total liabilities)/book value of total assets

2) To investigate the relationship between market based and accounting based performances: the relationship between accounting based and market based performances of banks in each emerging market will be tested to see whether the two types of performance measures have the positive correlation. The following GLS models with White Heteroskedasticity-Consistent Standard Errors \& Covariance is used to test the relationship: 
$\mathrm{Q}_{\mathrm{i}}=\alpha+\beta \mathrm{NIM}_{\mathrm{i}}+\varepsilon_{\mathrm{i}}$

$\mathrm{Q}_{\mathrm{i}}=\alpha+\beta \mathrm{ROA}_{\mathrm{i}}+\varepsilon_{\mathrm{i}}$

$\mathrm{Q}_{\mathrm{i}}=\alpha+\beta \mathrm{ROE}_{\mathrm{i}}+\varepsilon_{\mathrm{i}}$

where the subscript " $\mathrm{i}$ " stands for bank observation $\mathrm{i}$.

It is expected that the coefficients of NIM, ROA and ROE are positive since the accounting based and market based performance measures must be consistent.

3) To explore the determinants of market based and accounting based performances:

two GLS (to eliminate the possible serial correlation) regression models (with White Heteroskedasticity-Consistent Standard Errors \& Covariance) are constructed as follows:

$\mathrm{ROA}_{\mathrm{i}}$ or $\mathrm{ROE}_{\mathrm{i}}$ or $\mathrm{NIM}_{\mathrm{i}}$ or $\mathrm{Q}_{\mathrm{i}}=\alpha+\beta_{1} \mathrm{LLR}_{-} \mathrm{GL}_{\mathrm{i}}+\beta_{2} \mathrm{E}_{-} \mathrm{TA}_{\mathrm{i}}+\beta_{3} \mathrm{NL}_{-} \mathrm{DEPST} \mathrm{T}_{\mathrm{i}}$

$+\beta_{4}$ LA_DEPST $_{\mathrm{i}}+\beta_{5-11}$ MarketDummies $+\varepsilon_{\mathrm{i}}$

$\mathrm{Q}_{\mathrm{i}}=\alpha+\beta_{1} \mathrm{LLR}_{-} \mathrm{GL}_{\mathrm{i}}+\beta_{2} \mathrm{E}_{-} \mathrm{TA} \mathrm{A}_{\mathrm{i}}+\beta_{3} \mathrm{NL}_{-} \mathrm{DEPST} \mathrm{i}_{\mathrm{i}}+\beta_{4} \mathrm{LA}_{-} \mathrm{DEPST}_{\mathrm{i}}$

$+\beta_{5-11}$ MarketDummies $+\beta_{12} \mathrm{NIM}_{\mathrm{i}}+\beta_{13} \mathrm{ROA}_{\mathrm{i}}+\beta_{14} \mathrm{ROE}_{\mathrm{i}}+\varepsilon_{\mathrm{i}}$

Model (4) intends to investigate the determinants of accounting based performance ROA, ROE and NIM, and market based performance Q. Since Q is also expected to be affected by NIM, ROA and ROE, therefore, model (5) adds these 3 more variables to study the determinants of $\mathrm{Q}$.

To study the possible factors that may determine the market based or accounting based performance of banks, seven independent variables are selected following Chunahachinda and Li (2011) to proxy different aspects of bank characteristics based on the widely used CAMEL rating system:

Variable 1) LLR_GL stands for the ratio of loan loss reserves to gross loan, it is the proxy for the asset quality of the bank. Higher ratio implies poor quality of the loan extended, but higher ratio could also imply the bank's conservativeness against loss.

Variable 2) E_TA stands for the ratio of equity to total assets and represents the capital adequacy or the financial leverage of the bank. Higher ratio means higher capital adequacy and lower financial leverage.

Variables 3) NL_DEPST stands for the ratio of net loans to deposits and short term funding and 4) LA_DEPST stands for the ratio of liquid assets to deposits and short term funding, both of them measure the liquidity of the bank. Higher ratios of NL_DEPST and lower ratios of LA_DEPST suggest lower liquidity of the bank.

Variables 5) NIM, 6) ROA and 7) ROE measure the earning ability of the bank.

Seven market dummies are added in the model when the regression is run on the pooled sample to control for the specific characteristics of each emerging market (Thailand is chosen as the base case) and deleted when the regression is run on bank observations of a single market.

Further, variable Ln(TotalAssets) will be added if the regression is run on banks of a single emerging market to control the size effect on performance. 


\section{Empirical results}

Table I shows the descriptive statistics of banks in eight Asian emerging markets over the period 2005-2010. It can be seen that the average performances are quite different among eight emerging markets. Banks in Indonesia have the highest average Q ratio of 1.14, ROA of 1.82 percent and NIM of 5.34 percent, whereas banks in China and India has the highest average ROE of 18.22 percent. Banks in Korea, Taiwan and Thailand have the lowest average Q ratio of 1.00. Banks in Taiwan also have the lowest average ROA of negative 0.57 percent, ROE of negative 7.80 percent and NIM of 1.97 percent. For the ratio of LoanLossReserve/GrossLoan, banks in Philippines have the highest level of 7.76 percent (indicating poor asset quality) whereas banks in Taiwan have the lowest level of 1.51 percent. Regarding the equity ratio, banks in Philippines have the highest level of 13.51 percent (indicating higher percentage of equity required to protect the bank from bankruptcy, which is consistent with the poor asset quality ratio mentioned above) whereas banks in India have the lowest level of 5.75 percent. Banks in Philippines also have the lowest level of NetLoans/DepositAndShortTermFunding ratio of 50.48 percent opposed to the highest level of 103.68 percent in Korea, which suggests the aggressive loan extending policy and relatively poor liquidity position in Korean banks. Banks in Indonesia have the highest level of LiquidAsstes/DepositAndShortTermFunding ratio of 31.91 percent whereas banks in India have the lowest level of only 11.02 percent indicating poor liquidity position in Indian banks. 
Table I: Descriptive statistics of banks in Asian emerging markets over the period 2005-2010

\begin{tabular}{|c|c|c|c|c|c|c|c|c|}
\hline & $\begin{array}{l}\text { Loan } \\
\text { Loss } \\
\text { Reserve } \\
\text { / Gross } \\
\text { Loans } \\
\%\end{array}$ & $\begin{array}{l}\text { Equity } \\
\text { / Total } \\
\text { Assets } \\
\%\end{array}$ & $\begin{array}{l}\text { Net } \\
\text { Loans / } \\
\text { Dep } \\
\text { And ST } \\
\text { Funding } \\
\%\end{array}$ & $\begin{array}{l}\text { Liquid } \\
\text { Assets / } \\
\text { Dep } \\
\text { And ST } \\
\text { Funding } \\
\%\end{array}$ & $\begin{array}{l}\mathrm{Q} \\
\text { ratio }\end{array}$ & $\begin{array}{l}\text { Net } \\
\text { Interest } \\
\text { Margin } \\
\%\end{array}$ & $\begin{array}{l}\text { Return } \\
\text { on } \\
\text { Assets } \\
\text { (ROA) } \\
\%\end{array}$ & $\begin{array}{l}\text { Return } \\
\text { on } \\
\text { Equity } \\
\text { (ROE) } \\
\%\end{array}$ \\
\hline \multicolumn{9}{|c|}{ Banks in Asian emerging markets (470 observations) } \\
\hline Mean & 3.81 & 9.60 & 71.98 & 24.38 & 1.05 & 3.83 & 1.06 & 9.94 \\
\hline $\begin{array}{l}\text { Median } \\
\text { Standard }\end{array}$ & 2.58 & 8.13 & 69.18 & 22.22 & 1.02 & 3.45 & 1.01 & 12.32 \\
\hline Deviation & 3.52 & 6.67 & 35.10 & 13.60 & 0.14 & 1.90 & 1.85 & 16.38 \\
\hline Minimum & 0.38 & 1.47 & 14.37 & 3.15 & 0.80 & -0.95 & -6.34 & -126.14 \\
\hline Maximum & 29.72 & 55.02 & 421.17 & 90.21 & 2.44 & 12.50 & 16.85 & 52.79 \\
\hline \multicolumn{9}{|c|}{ Banks in China (64 observations) } \\
\hline Mean & 2.25 & 5.83 & 56.94 & 26.74 & 1.02 & 2.69 & 1.01 & 18.22 \\
\hline $\begin{array}{l}\text { Median } \\
\text { Standard }\end{array}$ & 2.22 & 5.79 & 55.99 & 26.15 & 1.01 & 2.63 & 1.08 & 18.04 \\
\hline Deviation & 0.67 & 2.12 & 6.21 & 9.37 & 0.05 & 0.33 & 0.31 & 5.31 \\
\hline Minimum & 0.71 & 2.24 & 41.72 & 4.52 & 0.93 & 2.03 & 0.14 & 4.18 \\
\hline Maximum & 4.00 & 13.07 & 70.09 & 44.24 & 1.16 & 3.48 & 1.73 & 31.17 \\
\hline \multicolumn{9}{|c|}{ Banks in India (29 observations) } \\
\hline Mean & 1.60 & 5.75 & 67.27 & 11.02 & 1.01 & 3.05 & 1.04 & 18.22 \\
\hline $\begin{array}{l}\text { Median } \\
\text { Standard }\end{array}$ & 1.50 & 5.60 & 67.80 & 11.10 & 1.01 & 2.92 & 1.02 & 17.05 \\
\hline Deviation & 0.76 & 1.07 & 5.56 & 2.47 & 0.03 & 0.58 & 0.38 & 5.78 \\
\hline Minimum & 0.38 & 4.13 & 52.40 & 3.15 & 0.96 & 1.91 & 0.38 & 8.03 \\
\hline Maximum & 3.81 & 8.49 & 78.59 & 15.85 & 1.06 & 4.30 & 1.68 & 27.98 \\
\hline \multicolumn{9}{|c|}{ Banks in Indonesia (133 observations) } \\
\hline Mean & 3.39 & 12.10 & 77.57 & 31.91 & 1.14 & 5.34 & 1.82 & 12.32 \\
\hline $\begin{array}{l}\text { Median } \\
\text { Standard }\end{array}$ & 2.58 & 9.64 & 70.71 & 27.86 & 1.09 & 5.27 & 1.22 & 11.49 \\
\hline Deviation & 3.07 & 8.85 & 54.40 & 15.60 & 0.21 & 2.28 & 2.74 & 10.39 \\
\hline Minimum & 0.81 & 2.76 & 20.26 & 6.68 & 0.87 & -0.95 & -1.19 & -54.95 \\
\hline Maximum & 29.72 & 55.02 & 421.17 & 90.21 & 2.44 & 12.50 & 16.85 & 35.89 \\
\hline \multicolumn{9}{|c|}{ Banks in Korea (41 observations) } \\
\hline Mean & 3.13 & 6.47 & 103.68 & 13.75 & 1.00 & 3.78 & 0.76 & 9.47 \\
\hline $\begin{array}{l}\text { Median } \\
\text { Standard }\end{array}$ & 2.08 & 6.23 & 90.31 & 11.15 & 0.99 & 3.41 & 0.86 & 13.98 \\
\hline Deviation & 2.77 & 1.70 & 36.56 & 5.93 & 0.03 & 1.46 & 1.38 & 30.04 \\
\hline Minimum & 1.37 & 1.47 & 68.01 & 5.60 & 0.92 & 1.50 & -3.54 & -126.14 \\
\hline Maximum & 13.40 & 12.09 & 213.29 & 30.38 & 1.07 & 10.39 & 3.70 & 52.79 \\
\hline
\end{tabular}




\begin{tabular}{|c|c|c|c|c|c|c|c|c|}
\hline & $\begin{array}{l}\text { Loan } \\
\text { Loss } \\
\text { Reserve } \\
\text { / Gross } \\
\text { Loans } \\
\% \\
\end{array}$ & $\begin{array}{l}\text { Equity } \\
/ \text { Total } \\
\text { Assets } \\
\%\end{array}$ & $\begin{array}{l}\text { Net } \\
\text { Loans / } \\
\text { Dep And } \\
\text { ST } \\
\text { Funding } \\
\% \\
\end{array}$ & $\begin{array}{l}\text { Liquid } \\
\text { Assets / } \\
\text { Dep And } \\
\text { ST } \\
\text { Funding } \\
\% \\
\end{array}$ & $\begin{array}{l}Q \\
\text { ratio }\end{array}$ & $\begin{array}{l}\text { Net } \\
\text { Interest } \\
\text { Margin } \\
\%\end{array}$ & $\begin{array}{l}\text { Return } \\
\text { on } \\
\text { Assets } \\
(\text { ROA) } \\
\% \\
\end{array}$ & $\begin{array}{l}\text { Return } \\
\text { on } \\
\text { Equity } \\
\text { (ROE) } \\
\% \\
\end{array}$ \\
\hline \multicolumn{9}{|c|}{ Banks in Malaysia (13 observations) } \\
\hline Mean & 3.34 & 7.49 & 63.59 & 30.08 & 1.07 & 2.80 & 1.10 & 14.87 \\
\hline $\begin{array}{l}\text { Median } \\
\text { Standard }\end{array}$ & 3.32 & 7.58 & 68.35 & 30.39 & 1.06 & 2.77 & 1.15 & 15.15 \\
\hline Deviation & 1.03 & 0.79 & 12.95 & 11.58 & 0.03 & 0.21 & 0.30 & 4.63 \\
\hline Minimum & 1.64 & 6.05 & 49.54 & 12.91 & 1.03 & 2.48 & 0.26 & 3.27 \\
\hline Maximum & 5.04 & 8.78 & 77.97 & 45.06 & 1.12 & 3.09 & 1.40 & 24.40 \\
\hline \multicolumn{9}{|c|}{ Banks in Philippines (72 observations) } \\
\hline Mean & 7.76 & 13.51 & 50.48 & 31.44 & 1.07 & 4.26 & 1.27 & 10.60 \\
\hline $\begin{array}{l}\text { Median } \\
\text { Standard }\end{array}$ & 6.92 & 11.19 & 50.86 & 29.07 & 1.03 & 3.88 & 1.20 & 11.03 \\
\hline Deviation & 4.72 & 6.84 & 16.15 & 12.75 & 0.15 & 1.17 & 0.74 & 5.64 \\
\hline Minimum & 1.34 & 6.90 & 14.37 & 12.19 & 0.93 & 2.87 & 0.14 & 0.40 \\
\hline Maximum & 23.85 & 40.49 & 87.72 & 76.54 & 1.58 & 7.75 & 4.58 & 32.54 \\
\hline \multicolumn{9}{|c|}{ Banks in Taiwan (61 observations) } \\
\hline Mean & 1.51 & 6.02 & 73.96 & 18.07 & 1.00 & 1.97 & -0.57 & -7.80 \\
\hline $\begin{array}{l}\text { Median } \\
\text { Standard }\end{array}$ & 1.19 & 5.63 & 76.08 & 17.40 & 1.00 & 1.43 & 0.07 & 1.31 \\
\hline Deviation & 1.15 & 2.24 & 8.38 & 8.04 & 0.03 & 1.29 & 1.55 & 22.17 \\
\hline Minimum & 0.38 & 2.75 & 53.81 & 5.71 & 0.88 & 0.75 & -5.55 & -101.31 \\
\hline Maximum & 6.76 & 15.74 & 91.08 & 33.42 & 1.06 & 7.06 & 1.12 & 15.23 \\
\hline \multicolumn{9}{|c|}{ Banks in Thailand (57 observations) } \\
\hline Mean & 5.75 & 11.57 & 82.40 & 15.18 & 1.00 & 3.73 & 1.05 & 8.28 \\
\hline $\begin{array}{l}\text { Median } \\
\text { Standard }\end{array}$ & 5.22 & 9.97 & 82.43 & 15.05 & 1.01 & 3.45 & 1.13 & 11.75 \\
\hline Deviation & 3.06 & 5.56 & 8.90 & 5.30 & 0.09 & 1.05 & 1.36 & 15.72 \\
\hline Minimum & 1.85 & 6.23 & 62.69 & 3.67 & 0.80 & 2.33 & -6.34 & -94.12 \\
\hline Maximum & 20.31 & 32.36 & 102.30 & 25.65 & 1.29 & 8.12 & 4.15 & 21.72 \\
\hline
\end{tabular}

Table II lists the correlation among variables. The correlations among variables are not too high to affect the regression results, thus no variables will be excluded from the model mentioned in the methodology section. It indicates that market based performance measure $Q$ is positively related to all accounting based performance measures: NIM, ROA and ROE. However, it can seen from Table III that Q ratio is found to be significantly positively related to only ROA and ROE (this is consistent with the expectation), but there is no significant relationship between Q and NIM although the coefficient is positive (this is not consistent with the expectation). This result could suggest that investors pay more attention on ROA and ROE rather than NIM to form their expectation on firm value. 
Table II: Correlations among variables

\begin{tabular}{|c|c|c|c|c|c|c|c|}
\hline & $\begin{array}{l}\text { Loan } \\
\text { Loss } \\
\text { Reserve } \\
\text { / Gross } \\
\text { Loans } \\
\%\end{array}$ & $\begin{array}{l}\text { Equity / } \\
\text { Total } \\
\text { Assets } \\
\%\end{array}$ & $\begin{array}{l}\text { Net } \\
\text { Interest } \\
\text { Margin } \\
\%\end{array}$ & $\begin{array}{l}\text { Return } \\
\text { on } \\
\text { Assets } \\
\text { (ROA) } \\
\%\end{array}$ & $\begin{array}{l}\text { Return } \\
\text { on } \\
\text { Equity } \\
\text { (ROE) } \\
\%\end{array}$ & $\begin{array}{l}\text { Net } \\
\text { Loans / } \\
\text { Dep } \\
\text { And ST } \\
\text { Fundin } \\
\text { g \% }\end{array}$ & $\begin{array}{l}\text { Liquid } \\
\text { Assets / } \\
\text { Dep } \\
\text { And ST } \\
\text { Fundin } \\
\text { g \% }\end{array}$ \\
\hline Equity / Total & & & & & & & \\
\hline $\begin{array}{l}\text { Assets \% } \\
\text { Net Interest Margin }\end{array}$ & 0.234 & & & & & & \\
\hline$\%$ & 0.132 & 0.190 & & & & & \\
\hline $\begin{array}{l}\text { Return on Assets } \\
(\text { ROA) \% }\end{array}$ & 0.112 & 0.641 & 0.051 & & & & \\
\hline $\begin{array}{l}\text { Return on Equity } \\
\text { (ROE) \% } \\
\text { Net Loans / Dep }\end{array}$ & -0.156 & 0.097 & 0.152 & 0.593 & & & \\
\hline $\begin{array}{l}\text { And ST Funding \% } \\
\text { Liquid Assets / Dep }\end{array}$ & -0.131 & 0.497 & -0.146 & 0.509 & 0.044 & & \\
\hline And ST Funding \% & 0.090 & 0.447 & 0.040 & 0.342 & 0.101 & 0.026 & \\
\hline Q ratio & -0.035 & 0.590 & 0.180 & 0.545 & 0.145 & 0.392 & 0.416 \\
\hline
\end{tabular}

Table III: Relationship between market based performance and accounting based performance (GLS with White Heteroskedasticity-Consistent Standard Errors \& Covariance) Dependent variable: $\mathrm{Q}$ ratio

\begin{tabular}{|c|c|c|c|c|c|}
\hline Independent & & Independent & & Independent & \\
\hline Variable & Coefficient & Variable & Coefficient & Variable & Coefficient \\
\hline $\mathrm{C}$ & $1.028 * * *$ & $\mathrm{C}$ & $1.011 * * *$ & $\mathrm{C}$ & $1.044^{* * *}$ \\
\hline & $(0.000)$ & & $(0.000)$ & & $(0.000)$ \\
\hline Net Interest & & Return & & Return & \\
\hline Margin & $\begin{array}{l}0.007 \\
(0.409)\end{array}$ & Assets & $\begin{array}{l}0.041^{* * *} \\
(0.000)\end{array}$ & Equity & $\begin{array}{l}0.001 * * * \\
(0.007)\end{array}$ \\
\hline R-squared & 0.049 & R-squared & 0.313 & R-squared & 0.058 \\
\hline Observations & 470 & Observations & 470 & Observations & 470 \\
\hline
\end{tabular}

Note: P-values are in parentheses. ${ }^{* * *},{ }^{* *}$ and $*$ indicate significance at $1 \%, 5 \%$ and $10 \%$ levels, respectively.

Table IV details the GLS regression results for the pooled sample, i.e. banks in the eight Asian emerging markets over the period 2005-2010. It is found that ROA is significantly positively related to the E_TA and NL_DEPST ratios. ROE is significantly negatively related to LLR_GL. NIM is significantly positively related to E_TA and negatively linked to NL_DEPST and LA_DEPST. Whereas the Q ratio is significantly positively related to E_TA and NL_DEPST and negatively linked to LLR_GL. 
Table IV: Results of GLS (with White Heteroskedasticity-Consistent Standard Errors \& Covariance) regressions for the pooled sample - banks in Asian emerging markets - over the period 2005-2010

\begin{tabular}{|c|c|c|c|c|c|}
\hline \multirow{3}{*}{$\begin{array}{l}\text { Independent } \\
\text { Variables }\end{array}$} & \multicolumn{5}{|c|}{ Dependent variable } \\
\hline & \multirow[t]{2}{*}{ ROA } & \multirow[t]{2}{*}{$\mathrm{ROE}$} & \multirow[t]{2}{*}{ NIM } & \multirow[t]{2}{*}{$\mathrm{Q}$} & \multirow[t]{2}{*}{$\mathrm{Q}$} \\
\hline & & & & & \\
\hline $\mathrm{C}$ & $\begin{array}{l}-2.146^{* * *} \\
(0.000)\end{array}$ & $\begin{array}{l}12.441^{* * *} \\
(0.000)\end{array}$ & $\begin{array}{l}5.301 * * * \\
(0.000)\end{array}$ & $\begin{array}{l}0.845^{* * *} \\
(0.000)\end{array}$ & $\begin{array}{l}0.889 * * * \\
(0.000)\end{array}$ \\
\hline LLR_GL & $\begin{array}{l}0.032 \\
(0.319)\end{array}$ & $\begin{array}{l}-1.347^{* * *} \\
(0.009)\end{array}$ & $\begin{array}{l}-0.038 \\
(0.247)\end{array}$ & $\begin{array}{l}-0.005 * * \\
(0.033)\end{array}$ & $\begin{array}{l}-0.009 * * * \\
(0.001)\end{array}$ \\
\hline E_TA & $\begin{array}{l}0.109 * * * \\
(0.002)\end{array}$ & $\begin{array}{l}0.140 \\
(0.430)\end{array}$ & $\begin{array}{l}0.088^{* * *} \\
(0.000)\end{array}$ & $\begin{array}{l}0.009 * * * \\
(0.005)\end{array}$ & $\begin{array}{l}0.005^{* *} \\
(0.041)\end{array}$ \\
\hline NL_DEPST & $\begin{array}{l}0.020 * * * \\
(0.000)\end{array}$ & $\begin{array}{l}0.028 \\
(0.180)\end{array}$ & $\begin{array}{l}-0.021^{* * *} \\
(0.000)\end{array}$ & $\begin{array}{l}0.001^{*} \\
(0.093)\end{array}$ & $\begin{array}{l}0.000 \\
(0.459)\end{array}$ \\
\hline LA_DEPST & $\begin{array}{l}0.008 \\
(0.182)\end{array}$ & $\begin{array}{l}-0.026 \\
(0.662)\end{array}$ & $\begin{array}{l}-0.043^{* * *} \\
(0.000)\end{array}$ & $\begin{array}{l}0.001^{*} \\
(0.079)\end{array}$ & $\begin{array}{l}0.001^{*} \\
(0.072)\end{array}$ \\
\hline CHINA & $\begin{array}{l}1.110 * * * \\
(0.000)\end{array}$ & $\begin{array}{l}7.109 * * * \\
(0.002)\end{array}$ & $\begin{array}{l}-0.703^{* * * *} \\
(0.003)\end{array}$ & $\begin{array}{l}0.054^{*} \\
(0.060)\end{array}$ & $\begin{array}{l}0.039 * \\
(0.070)\end{array}$ \\
\hline INDIA & $\begin{array}{l}1.092 * * * \\
(0.000)\end{array}$ & $\begin{array}{l}5.551 * * \\
(0.040)\end{array}$ & $\begin{array}{l}-0.826^{* * *} \\
(0.000)\end{array}$ & $\begin{array}{l}0.049 * \\
(0.054)\end{array}$ & $\begin{array}{l}0.033^{*} \\
(0.087)\end{array}$ \\
\hline INDONESIA & $\begin{array}{l}0.756 * * * \\
(0.001)\end{array}$ & $\begin{array}{l}1.423 \\
(0.528)\end{array}$ & $\begin{array}{l}2.088^{* * * *} \\
(0.000)\end{array}$ & $\begin{array}{l}0.101^{* * *} \\
(0.000)\end{array}$ & $\begin{array}{l}0.059 * * \\
(0.010)\end{array}$ \\
\hline KOREA & $\begin{array}{l}-0.060 \\
(0.875)\end{array}$ & $\begin{array}{l}-2.178 \\
(0.689)\end{array}$ & $\begin{array}{l}0.768^{* *} \\
(0.015)\end{array}$ & $\begin{array}{l}0.009 \\
(0.719)\end{array}$ & $\begin{array}{l}0.000 \\
(0.985)\end{array}$ \\
\hline MALAYSIA & $\begin{array}{l}0.844 * * * \\
(0.001)\end{array}$ & $\begin{array}{l}4.890 * * \\
(0.047)\end{array}$ & $\begin{array}{l}-0.421^{*} \\
(0.064)\end{array}$ & $\begin{array}{l}0.089 * * * \\
(0.001)\end{array}$ & $\begin{array}{l}0.074 * * * \\
(0.000)\end{array}$ \\
\hline PHILIPPINE & & & & & \\
\hline $\mathrm{S}$ & $\begin{array}{l}0.451^{*} \\
(0.077)\end{array}$ & $\begin{array}{l}6.141^{* *} \\
(0.027)\end{array}$ & $\begin{array}{l}0.477^{*} \\
(0.052)\end{array}$ & $\begin{array}{l}0.072 * * * \\
(0.008)\end{array}$ & $\begin{array}{l}0.066^{* *} \\
(0.016)\end{array}$ \\
\hline TAIWAN & $\begin{array}{l}-0.729 * * \\
(0.049)\end{array}$ & $\begin{array}{l}-20.626^{* * *} \\
(0.000)\end{array}$ & $\begin{array}{l}-1.486^{* * * *} \\
(0.000)\end{array}$ & $\begin{array}{l}0.023 \\
(0.369)\end{array}$ & $\begin{array}{l}0.020 \\
(0.305)\end{array}$ \\
\hline NIM & & & & & $\begin{array}{l}0.010^{* *} \\
(0.033)\end{array}$ \\
\hline ROA & & & & & $\begin{array}{l}0.033^{*} \\
(0.072)\end{array}$ \\
\hline ROE & & & & & $\begin{array}{l}-0.002^{* *} \\
(0.046)\end{array}$ \\
\hline R-squared & 0.563 & 0.278 & 0.492 & 0.471 & 0.508 \\
\hline Observations & 470 & 470 & 470 & 470 & 470 \\
\hline
\end{tabular}

Note: ROA stands for return on assets; ROE stands for return on equity; NIM stands for net interest margin; Q stands for Tobin's Q ratio; LLR_GL stands for loan loss reserve/gross loans; E_TA stands for equity/total assets; NL_DEPST stands for net loans/deposit and shortterm funding; LA_DEPST stands for liquid assets/deposits and short-term funding. P-values are in parentheses. ${ }^{* * *}, * *$ and $*$ indicate significance at $1 \%, 5 \%$ and $10 \%$ levels, respectively. 
Table V - XI list the GLS regression results for banks in seven emerging markets (except Malaysia because the number of observations is not sufficient to run the regression) over the period 2005-2010. The different regression results for banks in each single emerging market indicate that the market based and accounting based performances of commercial banks in different emerging market are affected by different factors in different ways. For instance, in China and Thailand, banks' ROA is positively affected by E_TA whereas Q ratio is negatively affected by E_TA. On the other hand, in Indonesia and Philippines, banks' Q ratio is positively affected by E_TA. In Indonesia, Korea and Taiwan, banks' ROE is negatively related to LLR_GL, but the Q ratios in these emerging markets are not affected by this factor at all.

Table V: Results of GLS (with White Heteroskedasticity-Consistent Standard Errors \& Covariance) regressions for the banks in China over the period 2005-2010

\begin{tabular}{|c|c|c|c|c|c|}
\hline \multirow{3}{*}{$\begin{array}{l}\text { Independent } \\
\text { Variables }\end{array}$} & \multicolumn{5}{|c|}{ Dependent variable } \\
\hline & ROA & ROE & NIM & $\mathrm{Q}$ & $\mathrm{Q}$ \\
\hline & & & & & \\
\hline $\mathrm{C}$ & $\begin{array}{l}0.337 \\
(0.501)\end{array}$ & $\begin{array}{l}8.514 \\
(0.400)\end{array}$ & $\begin{array}{l}3.893 * * * \\
(0.000)\end{array}$ & $\begin{array}{l}1.451^{* * *} \\
(0.000)\end{array}$ & $\begin{array}{l}1.462^{* * *} \\
(0.000)\end{array}$ \\
\hline LLR_GL & $\begin{array}{l}-0.089 \\
(0.121)\end{array}$ & $\begin{array}{l}-0.983 \\
(0.526)\end{array}$ & $\begin{array}{l}0.012 \\
(0.796)\end{array}$ & $\begin{array}{l}-0.010 \\
(0.224)\end{array}$ & $\begin{array}{l}-0.009 \\
(0.131)\end{array}$ \\
\hline E_TA & $\begin{array}{l}0.094^{* * *} \\
(0.000)\end{array}$ & $\begin{array}{l}-0.655^{*} \\
(0.074)\end{array}$ & $\begin{array}{l}0.023 \\
(0.294)\end{array}$ & $\begin{array}{l}-0.010 * * * \\
(0.000)\end{array}$ & $\begin{array}{l}-0.004 \\
(0.454)\end{array}$ \\
\hline NL_DEPST & $\begin{array}{l}-0.008 * * \\
(0.039)\end{array}$ & $\begin{array}{l}-0.033 \\
(0.746)\end{array}$ & $\begin{array}{l}0.002 \\
(0.792)\end{array}$ & $\begin{array}{l}-0.002 * * \\
(0.011)\end{array}$ & $\begin{array}{l}-0.002 * * \\
(0.017)\end{array}$ \\
\hline LA_DEPST & $\begin{array}{l}0.000 \\
(0.975)\end{array}$ & $\begin{array}{l}0.129 * \\
(0.071)\end{array}$ & $\begin{array}{l}-0.017 * * * \\
(0.000)\end{array}$ & $\begin{array}{l}-0.002 * * \\
(0.010)\end{array}$ & $\begin{array}{l}-0.003 * * * \\
(0.002)\end{array}$ \\
\hline LN_TA & $\begin{array}{l}0.056^{* * *} \\
(0.008)\end{array}$ & $\begin{array}{l}1.001 * * \\
(0.043)\end{array}$ & $\begin{array}{l}-0.071^{*} \\
(0.081)\end{array}$ & $\begin{array}{l}-0.012 * * * \\
(0.009)\end{array}$ & $\begin{array}{l}-0.014 * * * \\
(0.003)\end{array}$ \\
\hline NIM & & & & & $\begin{array}{l}-0.009 \\
(0.666)\end{array}$ \\
\hline ROA & & & & & $\begin{array}{l}-0.029 \\
(0.532)\end{array}$ \\
\hline ROE & & & & & $\begin{array}{l}0.003 * \\
(0.085)\end{array}$ \\
\hline $\begin{array}{l}\text { R-squared } \\
\text { Observation }\end{array}$ & 0.534 & 0.198 & 0.456 & 0.300 & 0.356 \\
\hline S & 64 & 64 & 64 & 64 & 64 \\
\hline
\end{tabular}

Note: ROA stands for return on assets; ROE stands for return on equity; NIM stands for net interest margin; Q stands for Tobin's Q ratio; LLR_GL stands for loan loss reserve/gross loans; E_TA stands for equity/total assets; NL_DEPST stands for net loans/deposit and shortterm funding; LA_DEPST stands for liquid assets/deposits and short-term funding; LN_TA stands for the natural logarithm of total assets. P-values are in parentheses. ${ }^{* * *}$, ${ }^{* *}$ and * indicate significance at $1 \%, 5 \%$ and $10 \%$ levels, respectively. 
Table VI: Results of GLS (with White Heteroskedasticity-Consistent Standard Errors \& Covariance) regressions for the banks in India over the period 2005-2010

\begin{tabular}{|c|c|c|c|c|c|}
\hline \multirow{3}{*}{$\begin{array}{l}\text { Independent } \\
\text { Variables }\end{array}$} & \multicolumn{5}{|c|}{ Dependent variable } \\
\hline & ROA & ROE & NIM & $\mathrm{Q}$ & $\mathrm{Q}$ \\
\hline & & & & & \\
\hline $\mathrm{C}$ & $\begin{array}{l}0.480 \\
(0.637)\end{array}$ & $\begin{array}{l}19.527 \\
(0.355)\end{array}$ & $\begin{array}{l}1.157 \\
(0.602)\end{array}$ & $\begin{array}{l}0.765^{* * *} \\
(0.000)\end{array}$ & $\begin{array}{l}0.798^{* * *} \\
(0.000)\end{array}$ \\
\hline LLR_GL & $\begin{array}{l}-0.012 \\
(0.924)\end{array}$ & $\begin{array}{l}0.715 \\
(0.756)\end{array}$ & $\begin{array}{l}0.420^{*} \\
(0.051)\end{array}$ & $\begin{array}{l}0.012 \\
(0.133)\end{array}$ & $\begin{array}{l}0.018^{*} \\
(0.093)\end{array}$ \\
\hline E_TA & $\begin{array}{l}0.228 * * * \\
(0.000)\end{array}$ & $\begin{array}{l}1.461 \\
(0.138)\end{array}$ & $\begin{array}{l}0.441^{* * *} \\
(0.001)\end{array}$ & $\begin{array}{l}0.005 \\
(0.214)\end{array}$ & $\begin{array}{l}-0.006 \\
(0.516)\end{array}$ \\
\hline NL_DEPST & $\begin{array}{l}-0.013 \\
(0.545)\end{array}$ & $\begin{array}{l}-0.161 \\
(0.677)\end{array}$ & $\begin{array}{l}-0.030 \\
(0.401)\end{array}$ & $\begin{array}{l}0.001 \\
(0.102)\end{array}$ & $\begin{array}{l}0.001 \\
(0.123)\end{array}$ \\
\hline LA_DEPST & $\begin{array}{l}0.045 \\
(0.108)\end{array}$ & $\begin{array}{l}0.930^{*} \\
(0.073)\end{array}$ & $\begin{array}{l}0.023 \\
(0.637)\end{array}$ & $\begin{array}{l}-0.002 \\
(0.367)\end{array}$ & $\begin{array}{l}-0.004^{*} \\
(0.050)\end{array}$ \\
\hline LN_TA & $\begin{array}{l}-0.026 \\
(0.703)\end{array}$ & $\begin{array}{l}-0.733 \\
(0.570)\end{array}$ & $\begin{array}{l}0.031 \\
(0.695)\end{array}$ & $\begin{array}{l}0.010^{* * *} \\
(0.002)\end{array}$ & $\begin{array}{l}0.013^{* * *} \\
(0.002)\end{array}$ \\
\hline NIM & & & & & $\begin{array}{l}-0.017 \\
(0.319)\end{array}$ \\
\hline ROA & & & & & $\begin{array}{l}0.075 \\
(0.239)\end{array}$ \\
\hline ROE & & & & & $\begin{array}{l}-0.002 \\
(0.531)\end{array}$ \\
\hline $\begin{array}{l}\text { R-squared } \\
\text { Observation }\end{array}$ & 0.542 & 0.247 & 0.526 & 0.456 & 0.573 \\
\hline $\mathrm{s}$ & 29 & 29 & 29 & 29 & 29 \\
\hline
\end{tabular}

Note: ROA stands for return on assets; ROE stands for return on equity; NIM stands for net interest margin; Q stands for Tobin's Q ratio; LLR_GL stands for loan loss reserve/gross loans; E_TA stands for equity/total assets; NL_DEPST stands for net loans/deposit and shortterm funding; LA_DEPST stands for liquid assets/deposits and short-term funding; LN_TA stands for the natural logarithm of total assets. P-values are in parentheses. ***, ** and * indicate significance at $1 \%, 5 \%$ and $10 \%$ levels, respectively. 
Table VII: Results of GLS (with White Heteroskedasticity-Consistent Standard Errors \& Covariance) regressions for the banks in Indonesia over the period 2005-2010

\begin{tabular}{|c|c|c|c|c|c|}
\hline \multirow{3}{*}{$\begin{array}{l} \\
\text { Independent } \\
\text { Variables }\end{array}$} & \multicolumn{5}{|c|}{ Dependent variable } \\
\hline & ROA & ROE & NIM & $\mathrm{Q}$ & $\mathrm{Q}$ \\
\hline & & & & & \\
\hline $\mathrm{C}$ & $\begin{array}{l}-7.806 * * * \\
(0.000)\end{array}$ & $\begin{array}{l}-59.195^{* * *} \\
(0.000)\end{array}$ & $\begin{array}{l}-1.254 \\
(0.372)\end{array}$ & $\begin{array}{l}0.484 * * * \\
(0.001)\end{array}$ & $\begin{array}{l}0.548^{* * *} \\
(0.000)\end{array}$ \\
\hline LLR_GL & $\begin{array}{l}0.069 \\
(0.227)\end{array}$ & $\begin{array}{l}-1.948^{* * *} \\
(0.000)\end{array}$ & $\begin{array}{l}-0.145^{* * *} \\
(0.001)\end{array}$ & $\begin{array}{l}-0.001 \\
(0.735)\end{array}$ & $\begin{array}{l}-0.006 \\
(0.467)\end{array}$ \\
\hline E_TA & $\begin{array}{l}0.226 * * * \\
(0.000)\end{array}$ & $\begin{array}{l}0.495^{* * * *} \\
(0.000)\end{array}$ & $\begin{array}{l}0.050 \\
(0.174)\end{array}$ & $\begin{array}{l}0.015^{*} \\
(0.054)\end{array}$ & $\begin{array}{l}0.009 * \\
(0.075)\end{array}$ \\
\hline NL_DEPST & $\begin{array}{l}0.006 \\
(0.343)\end{array}$ & $\begin{array}{l}-0.030 * * \\
(0.046)\end{array}$ & $\begin{array}{l}-0.019 * * * \\
(0.000)\end{array}$ & $\begin{array}{l}0.000 \\
(0.789)\end{array}$ & $\begin{array}{l}0.000 \\
(0.837)\end{array}$ \\
\hline LA_DEPST & $\begin{array}{l}0.017 * * \\
(0.025)\end{array}$ & $\begin{array}{l}0.079 * * \\
(0.025)\end{array}$ & $\begin{array}{l}-0.048^{* * * *} \\
(0.001)\end{array}$ & $\begin{array}{l}0.001 \\
(0.540)\end{array}$ & $\begin{array}{l}0.001 \\
(0.401)\end{array}$ \\
\hline LN_TA & $\begin{array}{l}0.329 * * * \\
(0.000)\end{array}$ & $\begin{array}{l}4.210 * * * \\
(0.000)\end{array}$ & $\begin{array}{l}0.554 * * * \\
(0.000)\end{array}$ & $\begin{array}{l}0.026 * * * \\
(0.001)\end{array}$ & $\begin{array}{l}0.021^{* *} \\
(0.032)\end{array}$ \\
\hline NIM & & & & & $\begin{array}{l}0.013^{* *} \\
(0.037)\end{array}$ \\
\hline ROA & & & & & $\begin{array}{l}0.028 \\
(0.418)\end{array}$ \\
\hline $\mathrm{ROE}$ & & & & & $\begin{array}{l}-0.002 \\
(0.347)\end{array}$ \\
\hline $\begin{array}{l}\text { R-squared } \\
\text { Observation }\end{array}$ & 0.869 & 0.686 & 0.408 & 0.536 & 0.555 \\
\hline $\mathrm{s}$ & 133 & 133 & 133 & 133 & 133 \\
\hline
\end{tabular}

Note: ROA stands for return on assets; ROE stands for return on equity; NIM stands for net interest margin; Q stands for Tobin's Q ratio; LLR_GL stands for loan loss reserve/gross loans; E_TA stands for equity/total assets; NL_DEPST stands for net loans/deposit and shortterm funding; LA_DEPST stands for liquid assets/deposits and short-term funding; LN_TA stands for the natural logarithm of total assets. P-values are in parentheses. ***, ** and * indicate significance at $1 \%, 5 \%$ and $10 \%$ levels, respectively. 
Table VIII: Results of GLS (with White Heteroskedasticity-Consistent Standard Errors \& Covariance) regressions for the banks in Korea over the period 2005-2010

\begin{tabular}{|c|c|c|c|c|c|}
\hline \multirow{3}{*}{$\begin{array}{l} \\
\text { Independent } \\
\text { Variables }\end{array}$} & \multicolumn{5}{|c|}{ Dependent variable } \\
\hline & ROA & ROE & NIM & $\mathrm{Q}$ & $\mathrm{Q}$ \\
\hline & & & & & \\
\hline $\mathrm{C}$ & $\begin{array}{l}0.001 \\
(1.000)\end{array}$ & $\begin{array}{l}-3.426 \\
(0.879)\end{array}$ & $\begin{array}{l}7.422 * * * \\
(0.008)\end{array}$ & $\begin{array}{l}0.856 * * * \\
(0.000)\end{array}$ & $\begin{array}{l}0.842^{* * *} \\
(0.000)\end{array}$ \\
\hline LLR_GL & $\begin{array}{l}-0.078 \\
(0.511)\end{array}$ & $\begin{array}{l}-9.221 * * * \\
(0.000)\end{array}$ & $\begin{array}{l}0.146 \\
(0.259)\end{array}$ & $\begin{array}{l}0.001 \\
(0.487)\end{array}$ & $\begin{array}{l}-0.002 \\
(0.498)\end{array}$ \\
\hline E_TA & $\begin{array}{l}0.062 \\
(0.752)\end{array}$ & $\begin{array}{l}7.491 * * * \\
(0.004)\end{array}$ & $\begin{array}{l}-0.101 \\
(0.620)\end{array}$ & $\begin{array}{l}-0.005 * * * \\
(0.001)\end{array}$ & $\begin{array}{l}-0.003 \\
(0.221)\end{array}$ \\
\hline NL_DEPST & $\begin{array}{l}-0.003 \\
(0.572)\end{array}$ & $\begin{array}{l}0.065 \\
(0.241)\end{array}$ & $\begin{array}{l}-0.005 \\
(0.156)\end{array}$ & $\begin{array}{l}0.000^{* * *} \\
(0.000)\end{array}$ & $\begin{array}{l}0.000^{* * *} \\
(0.001)\end{array}$ \\
\hline LA_DEPST & $\begin{array}{l}-0.060 \\
(0.105)\end{array}$ & $\begin{array}{l}-1.540^{* *} \\
(0.034)\end{array}$ & $\begin{array}{l}-0.051 \\
(0.136)\end{array}$ & $\begin{array}{l}-0.001^{* *} \\
(0.018)\end{array}$ & $\begin{array}{l}-0.002 * * * \\
(0.006)\end{array}$ \\
\hline LN_TA & $\begin{array}{l}0.104 \\
(0.517)\end{array}$ & $\begin{array}{l}0.426 \\
(0.744)\end{array}$ & $\begin{array}{l}-0.137 \\
(0.409)\end{array}$ & $\begin{array}{l}0.014^{* * *} \\
(0.000)\end{array}$ & $\begin{array}{l}0.013 * * * \\
(0.000)\end{array}$ \\
\hline NIM & & & & & $\begin{array}{l}0.004 \\
(0.217)\end{array}$ \\
\hline ROA & & & & & $\begin{array}{l}0.001 \\
(0.727)\end{array}$ \\
\hline ROE & & & & & $\begin{array}{l}0.000 \\
(0.187)\end{array}$ \\
\hline $\begin{array}{l}\text { R-squared } \\
\text { Observation }\end{array}$ & 0.367 & 0.700 & 0.153 & 0.708 & 0.758 \\
\hline $\mathrm{s}$ & 41 & 41 & 41 & 41 & 41 \\
\hline
\end{tabular}

Note: ROA stands for return on assets; ROE stands for return on equity; NIM stands for net interest margin; Q stands for Tobin's Q ratio; LLR_GL stands for loan loss reserve/gross loans; E_TA stands for equity/total assets; NL_DEPST stands for net loans/deposit and shortterm funding; LA_DEPST stands for liquid assets/deposits and short-term funding; LN_TA stands for the natural logarithm of total assets. P-values are in parentheses. ***, ** and * indicate significance at $1 \%, 5 \%$ and $10 \%$ levels, respectively. 
Table IX: Results of GLS (with White Heteroskedasticity-Consistent Standard Errors \& Covariance) regressions for the banks in Philippines over the period 2005-2010

\begin{tabular}{|c|c|c|c|c|c|}
\hline \multirow{3}{*}{$\begin{array}{l} \\
\text { Independent } \\
\text { Variables }\end{array}$} & \multicolumn{5}{|c|}{ Dependent variable } \\
\hline & ROA & ROE & NIM & $\mathrm{Q}$ & $\mathrm{Q}$ \\
\hline & & & & & \\
\hline $\mathrm{C}$ & $\begin{array}{l}-2.049 \\
(0.234)\end{array}$ & $\begin{array}{l}-0.712 \\
(0.957)\end{array}$ & $\begin{array}{l}-0.326 \\
(0.845)\end{array}$ & $\begin{array}{l}1.727 * * * \\
(0.000)\end{array}$ & $\begin{array}{l}1.696 * * * \\
(0.000)\end{array}$ \\
\hline LLR_GL & $\begin{array}{l}0.003 \\
(0.908)\end{array}$ & $\begin{array}{l}-0.109 \\
(0.573)\end{array}$ & $\begin{array}{l}0.086^{* * *} \\
(0.000)\end{array}$ & $\begin{array}{l}-0.018^{* * *} \\
(0.000)\end{array}$ & $\begin{array}{l}-0.018 * * * \\
(0.002)\end{array}$ \\
\hline E_TA & $\begin{array}{l}0.025 \\
(0.535)\end{array}$ & $\begin{array}{l}-0.169 \\
(0.583)\end{array}$ & $\begin{array}{l}0.074 * * \\
(0.046)\end{array}$ & $\begin{array}{l}0.011 * * \\
(0.034)\end{array}$ & $\begin{array}{l}0.011 \\
(0.144)\end{array}$ \\
\hline NL_DEPST & $\begin{array}{l}0.002 \\
(0.807)\end{array}$ & $\begin{array}{l}0.005 \\
(0.940)\end{array}$ & $\begin{array}{l}0.052^{* * *} \\
(0.000)\end{array}$ & $\begin{array}{l}-0.004^{* *} \\
(0.019)\end{array}$ & $\begin{array}{l}-0.004 * * \\
(0.037)\end{array}$ \\
\hline LA_DEPST & $\begin{array}{l}0.003 \\
(0.824)\end{array}$ & $\begin{array}{l}0.013 \\
(0.891)\end{array}$ & $\begin{array}{l}0.000 \\
(0.975)\end{array}$ & $\begin{array}{l}-0.001 \\
(0.461)\end{array}$ & $\begin{array}{l}-0.001 \\
(0.470)\end{array}$ \\
\hline LN_TA & $\begin{array}{l}0.233 * * \\
(0.048)\end{array}$ & $\begin{array}{l}1.167 \\
(0.202)\end{array}$ & $\begin{array}{l}0.024 \\
(0.837)\end{array}$ & $\begin{array}{l}-0.038 * * \\
(0.039)\end{array}$ & $\begin{array}{l}-0.038^{*} \\
(0.051)\end{array}$ \\
\hline NIM & & & & & $\begin{array}{l}0.009 \\
(0.724)\end{array}$ \\
\hline ROA & & & & & $\begin{array}{l}-0.027 \\
(0.703)\end{array}$ \\
\hline ROE & & & & & $\begin{array}{l}0.004 \\
(0.657)\end{array}$ \\
\hline $\begin{array}{l}\text { R-squared } \\
\text { Observation }\end{array}$ & 0.163 & 0.281 & 0.811 & 0.611 & 0.615 \\
\hline $\mathrm{s}$ & 72 & 72 & 72 & 72 & 72 \\
\hline
\end{tabular}

Note: ROA stands for return on assets; ROE stands for return on equity; NIM stands for net interest margin; Q stands for Tobin's Q ratio; LLR_GL stands for loan loss reserve/gross loans; E_TA stands for equity/total assets; NL_DEPST stands for net loans/deposit and shortterm funding; LA_DEPST stands for liquid assets/deposits and short-term funding; LN_TA stands for the natural logarithm of total assets. P-values are in parentheses. ${ }^{* * *}$, ** and * indicate significance at $1 \%, 5 \%$ and $10 \%$ levels, respectively. 
Table X: Results of GLS (with White Heteroskedasticity-Consistent Standard Errors \& Covariance) regressions for the banks in Taiwan over the period 2005-2010

\begin{tabular}{|c|c|c|c|c|c|}
\hline \multirow{3}{*}{$\begin{array}{l} \\
\text { Independent } \\
\text { Variables }\end{array}$} & \multicolumn{5}{|c|}{ Dependent variable } \\
\hline & ROA & ROE & NIM & $\mathrm{Q}$ & $\mathrm{Q}$ \\
\hline & & & & & \\
\hline $\mathrm{C}$ & $\begin{array}{l}1.303 \\
(0.613)\end{array}$ & $\begin{array}{l}-26.023 \\
(0.522)\end{array}$ & $\begin{array}{l}4.132 * * \\
(0.043)\end{array}$ & $\begin{array}{l}1.129 * * * \\
(0.000)\end{array}$ & $\begin{array}{l}1.057 * * * \\
(0.000)\end{array}$ \\
\hline LLR_GL & $\begin{array}{l}-0.959 * * * \\
(0.000)\end{array}$ & $\begin{array}{l}-13.615^{* * *} \\
(0.000)\end{array}$ & $\begin{array}{l}0.651^{* * *} \\
(0.000)\end{array}$ & $\begin{array}{l}0.003 \\
(0.373)\end{array}$ & $\begin{array}{l}0.004 \\
(0.267)\end{array}$ \\
\hline E_TA & $\begin{array}{l}-0.053 \\
(0.507)\end{array}$ & $\begin{array}{l}2.108 * * \\
(0.036)\end{array}$ & $\begin{array}{l}0.055 \\
(0.144)\end{array}$ & $\begin{array}{l}-0.010^{* * *} \\
(0.001)\end{array}$ & $\begin{array}{l}-0.006 \\
(0.103)\end{array}$ \\
\hline NL_DEPST & $\begin{array}{l}-0.015 \\
(0.434)\end{array}$ & $\begin{array}{l}-0.342 \\
(0.304)\end{array}$ & $\begin{array}{l}-0.043 * * * \\
(0.000)\end{array}$ & $\begin{array}{l}0.000 \\
(0.588)\end{array}$ & $\begin{array}{l}0.000 \\
(0.694)\end{array}$ \\
\hline LA_DEPST & $\begin{array}{l}-0.001 \\
(0.936)\end{array}$ & $\begin{array}{l}-0.043 \\
(0.888)\end{array}$ & $\begin{array}{l}-0.031 * * * \\
(0.004)\end{array}$ & $\begin{array}{l}0.000 \\
(0.757)\end{array}$ & $\begin{array}{l}0.000 \\
(0.423)\end{array}$ \\
\hline LN_TA & $\begin{array}{l}0.082 \\
(0.681)\end{array}$ & $\begin{array}{l}4.009 \\
(0.231)\end{array}$ & $\begin{array}{l}0.046 \\
(0.724)\end{array}$ & $\begin{array}{l}-0.005 \\
(0.235)\end{array}$ & $\begin{array}{l}-0.002 \\
(0.702)\end{array}$ \\
\hline NIM & & & & & $\begin{array}{l}0.002 \\
(0.625)\end{array}$ \\
\hline ROA & & & & & $\begin{array}{l}0.018 \\
(0.119)\end{array}$ \\
\hline ROE & & & & & $\begin{array}{l}-0.001 \\
(0.105)\end{array}$ \\
\hline $\begin{array}{l}\text { R-squared } \\
\text { Observation }\end{array}$ & 0.543 & 0.374 & 0.697 & 0.428 & 0.488 \\
\hline $\mathrm{s}$ & 61 & 61 & 61 & 61 & 61 \\
\hline
\end{tabular}

Note: ROA stands for return on assets; ROE stands for return on equity; NIM stands for net interest margin; Q stands for Tobin's Q ratio; LLR_GL stands for loan loss reserve/gross loans; E_TA stands for equity/total assets; NL_DEPST stands for net loans/deposit and shortterm funding; LA_DEPST stands for liquid assets/deposits and short-term funding; LN_TA stands for the natural logarithm of total assets. P-values are in parentheses. ***, ** and * indicate significance at $1 \%, 5 \%$ and $10 \%$ levels, respectively. 
Table XI: Results of GLS (with White Heteroskedasticity-Consistent Standard Errors \& Covariance) regressions for the banks in Thailand over the period 2005-2010

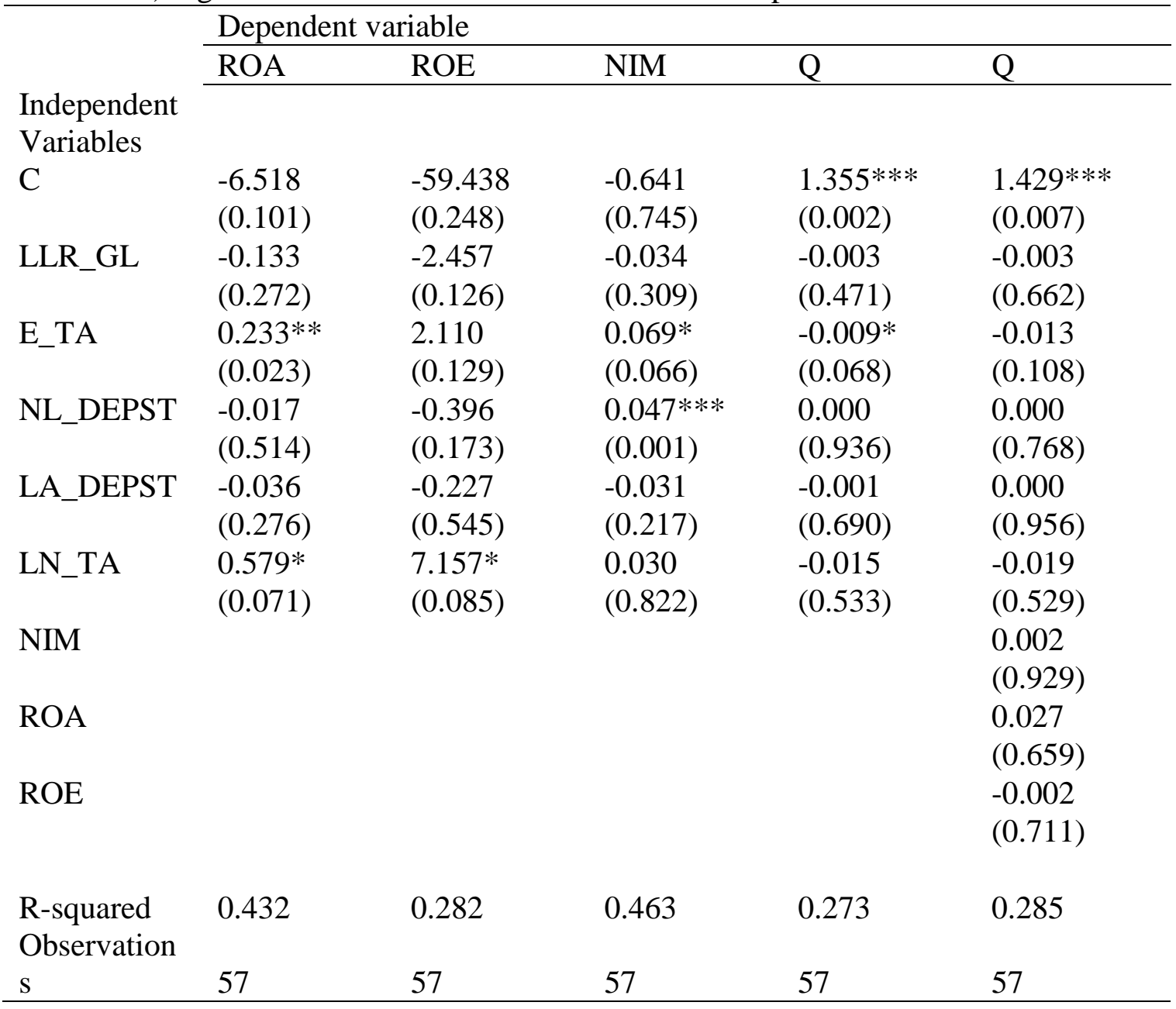

Note: ROA stands for return on assets; ROE stands for return on equity; NIM stands for net interest margin; Q stands for Tobin's Q ratio; LLR_GL stands for loan loss reserve/gross loans; E_TA stands for equity/total assets; NL_DEPST stands for net loans/deposit and shortterm funding; LA_DEPST stands for liquid assets/deposits and short-term funding; LN_TA stands for the natural logarithm of total assets. P-values are in parentheses. ***, ** and * indicate significance at $1 \%, 5 \%$ and $10 \%$ levels, respectively.

\section{Conclusion}

The study focuses on the comparison of the market based and accounting based performances of banks among eight Asian emerging markets over the period 2005-2010.

Market based performance is measured by Tobin's Q and accounting based performance is measured by three traditional ratios for a bank: NIM, ROA and ROE. The results show that the average performances are quite different among eight emerging markets. Banks in Indonesia have the highest average Q ratio of 1.14, ROA of 1.82 percent and NIM of 5.34 percent, whereas banks in China and India has the highest average ROE of 18.22 percent. Overall, Q ratio is found to be significantly positively related to ROA and ROE, but there is no significant relationship between Q and NIM. 
The different regression results for banks in each single emerging market indicate that the market based and accounting based performances of commercial banks in different emerging market are affected by different factors in different ways. The reason for this could be that different accounting practices or different banking regulations are adopted in different markets. For instance, Packer and Zhu (2012) find that banks in Southeast Asia with low credit quality are more likely to adopt income smoothing and procyclical loan loss provisions, whereas banks in India have the most striking countercyclical loan loss provisioning. However, this investigation is beyond the scope of this paper. Hence, it is of great interest to have the further research on this issue.

To sum up, although the eight economies are in the same region (Asia) and all named emerging markets, the market based and accounting based performances of commercial banks in each economy can be very different and the determinants of each type of performance are different. All of these findings will be useful for the banks' managers and their authorities to find out how good their performance is when comparing to other Asian emerging markets. Further, they will learn what factors tend to affect their market based performance and what factors tend to affect their accounting based performance, which will consequently suggest the directions to improve themselves.

\section{References}

Berger, A. N., Clarke, G.R.G., Cull, R., Klapper, L. and Udell, G.F., (2005), “Corporate governance and bank performance: A joint analysis of the static, selection, and dynamic effects of domestic, foreign, and state ownership”, Journal of Banking and Finance, vol. 29, pp. 2179-2221.

Berger, A. N., Deyoung, R., Genay, H. and Udell, G.F, (2000), "Globalization of financial institutions: Evidence from cross-border banking performance”, Brookings-Wharton Papers on Financial Services, vol. 3, pp. 23-158.

Bonin, J. P., Hasan, I., and Wachtel, P., (2005), "Bank performance, efficiency and ownership in transition countries”, Journal of Banking and Finance, vol. 29, pp. 31-53.

Choi, S., and Hasan, I., (2005), "Ownership, governance, and bank performance: Korean experience”, Financial Markets, Institutions and Instruments, vol. 14, pp. 215-241.

Chunhachinda, P. and Jumreornvong, S., (1999), "Competitiveness of banks and finance companies in Thailand”, Thammasat Review, vol. 4, no. 1, pp. 59-88.

Chunhachinda, P. and Li, L., (2011), “Competitiveness of Asian banks after recovering from the 1997 financial crisis”, working paper.

Joen, B. N., Olivero, M. P. and Wu J., (2010), "Do foreign banks increase competition? Evidence from emerging Asian and Latin American banking markets", Journal of Banking and Finance, doi:10.1016/j.jbankfin.2010.10.012.

Jones, J. S., Miller, S. A. and Yeager, T. J., (2011), “Charter value, Tobin's Q and bank risk during the subprime financial crisis”, Journal of Economics and Business, vol. 63, pp. 372-391. 
Jonghe, O. D. and Vennet, R. V., (2008), "Competition versus efficiency: What drives franchise values in european banking?” Journal of Banking and Finance, vol. 32, pp. 1820-1835.

Kosmidou, K. and Zopounidis, C., (2008), “Measurement of bank performance in Greece”, South-Eastern Europe Journal of Economics, vol. 1, pp. 79-95.

Lin, X. and Zhang, Y., (2009), "Bank ownership reform and bank performance in China”, Journal of Banking and Finance, vol. 33, pp. 22-29.

Micco, A. Panizza, U. and Yanez, M., (2007), "Bank ownership and performance: Does politics matter?” Journal of Banking and Finance, vol. 31, pp. 219-241.

Packer, F. and Zhu, H. B., (2012), “Loan loss provisioning practices of Asian banks”, BIS Working Papers, No. 375. 